\title{
A Chinese perspective on factors and molecular mechanisms that influence the curative effects of platelet transfusion
}

\author{
Meng Gao, Rong Huang, Hao Tang, Yunfeng Fu, Fengxia Liu, Rong Gui * \\ The Third Xiangya Hospital, Central South University, Changsha 410013, China.
}

\begin{abstract}
Platelet transfusion, as the primary treatment for severely low platelet count (thrombocytopenia) or disorders of platelet function, is used extensively in patients with blood system diseases or active hemorrhaging. In recent decades platelet related researches in China have steadily increased. In the clinical application of platelet transfusion, there are large differences between the treatment's effects on different patients. Immune or nonimmune factors, such as platelet-specific antibodies, platelet gene analysis and matches, and auxiliary treatment, may affect the curative effect, and even result in ineffective platelet transfusion. Platelets used in clinical therapy include platelets made from whole blood and hemapheresis platelets, and the latter is the main application type used in China. Both preparation and preservation methods can affect the quality of platelets and influence the therapeutic effect. Researches on the molecular basis or the curative effect of platelet transfusion are increasing, such as the relationship between platelet-activating factors, tissue factors, thrombopoietin, membrane glycoprotein and platelet activation, and their influence on the curative effect of platelet transfusion, the inhibition of antiplatelet therapy in platelet activation, and platelet signal transduction pathways and their significance in the curative effect of platelet transfusion. This article aims to review the research progress on the curative effect of platelet transfusion and its molecular mechanisms.
\end{abstract}

Keywords: platelet transfusion, effect factors, molecular mechanisms

\section{INTRODUCTION}

Since the 1950s, platelet transfusion has been widely used in the clinical treatment of patients with thrombocytopenia, and can effectively reduce mortality due to hemorrhaging. Platelet transfusion aims to maintain the platelet count within a safe range. However, about $30 \%-70 \%$ of patients fail to reach the expected level after transfusion, with some displaying platelet transfusion refractoriness (PTR). These patients usually require multiple platelet transfusions, which inevitably increases the economic and physical burden on them. PTR has become a clinical problem,

*Correspondence to: Dr. Rong Gui, Department of Blood Transfusion, The Third Xiangya Hospital, Central South University, Changsha, 410013 China. which is difficult to overcome, being one of the most widely studied research fields according to relevant literature, and Chinese scholars also have done a lot of researches in this field. This article intends to review the influence factors of platelet transfusion and to discuss the molecular basis and mechanisms of platelet related diseases.

\section{THE EFFECT FACTORS OF PLATELET TRANSFUSION}

In the clinical application of platelet transfusion, there is a considerable difference on the therapeutic effects among different patients, and some of the patients even display PTR, a challenging clinical problem, which is still difficult to overcome ${ }^{[1]}$. The influence factors of PTR generally include immune and 
non-immune factors ${ }^{[2]}$. The immune factor mainly refers to alloimmune response caused by repeated blood transfusions, which induces antiplatelet antibodies in the patient' plasma, and then quickly destroys the transfused platelets. Non-immune factors usually include fever, infection, liver and spleen enlargement, drugs, quality and quantity of platelets, patient's condition, etc.

\section{The preparation and preservation of platelets}

Preparation and preservation methods of platelets are closely related to their quality. The platelet components used in clinical therapy mainly include apheresis platelets (APs) and platelet concentrates (PCs). Since the 1980's, PCs have appeared along with the development of automation instruments, which have increased the supply of platelets ${ }^{[3]}$. Several studies have discussed the treatment effects of APs and PCs, but as yet the results are contradictory, and may be unequal for different diseases. Priority is given to the former in China. One research has shown that APs have good therapeutic effects on hematologic diseases ${ }^{[4]}$. Because of the increased requests for platelet products, APs obviously can't completely satisfy clinical requirements thus the application of concentrated platelets has increased. Studies indicate that PCs have higher counts and CCI values than APs, and clinical observation also supports that the effects of PCs are close to $\mathrm{APs}^{[5,6]}$. The preparation methods of PCs include platelet-rich plasma (PRP) and buffy coat (BC), and the latter method was dated from the 1980s. A high centrifugal force is used on whole blood to separate $\mathrm{BC}$, then lightly to remove red blood cells (RBCs) and white blood cells (WBCs) and obtain PCs. BC was widely used in European countries, more than $85 \%$ are BC. At present, platelets are conventionally stored at 22 for a maximum of 5 days, and the time constraint seriously limits its clinical application. Frozen platelets stored at -80 have the advantage of a long term storage time and thus large stocks can be accumulated. Although the effects of frozen platelets are not as beneficial as for fresh supplies, they can still be used for emergency cases ${ }^{[7]}$. Studies show no statistically significant difference in BPC, PDW, MPV, P-LCR and $\mathrm{pH}$ between frozen platelets stored for 6 months and fresh platelets, concluding that they are both effective on the treatment of acute hemorrhagic disease ${ }^{[8,9]}$.

\section{Human platelet antigen and human leukocyte antigen}

Platelet antigen can be divided into two types according to its distribution: 1) antigens common to other cells or tissues, such as the ABO blood group antigen, human leukocyte antigen (HLA)-I, etc. 2) platelet specific antigen namely human platelet antigen (HPA). The alloantibody may be induced in the process of platelet transfusion or pregnancy if the platelet antigen is not matched ${ }^{[10]}$, and cause immune diseases such as neonatal immune thrombocytopenic purpura (ITP) or post-transfusion purpura (PTA) and PTR.HLA antibody caused by repeatedly incompatible platelet transfusion is the main reason for alloimmunization. Studies on hematopoietic stem cell transplantation and haematological diseases in children have shown that HLA - I semi-compatible or compatible platelets can obviously enhance the therapeutic effects on PTR patients ${ }^{[11]}$. The HPA genotyping method is based on polymerase chain reaction (PCR) ${ }^{[12]}$. With the indepth study of HPA and the incessant development of gene detection technology, research on HPA plays a significant role in clinical and blood transfusion practice $^{[13]}$. The HPA-matched test can select compatible platelets for patients, and may reduce the occurrence of PTR. Since the distribution of HPA differs in ethnicities and regions, it is particularly important to establish local platelet gene banks ${ }^{[14]}$. Local blood donor HPA gene banks will also reduce patients' medical costs and find matched platelets quickly ${ }^{[15]}$. Several studies have reported HPA gene frequency and distribution characteristics in Chinese population ${ }^{[16-20]}$. Liu et $a l^{[21]}$ administered HPA genotyping of PTR patients and selected compatible platelets, which resulted in an improved outcome. Studies indicate that the transfusion of platelets compatible with the ABO blood group antigen, HLA and HPA type, is the best way to solve platelet immunity correlated diseases ${ }^{[22]}$.

\section{Threshold of platelet transfusion}

The clinical application of platelets includes both therapeutic and prophylactic transfusions. The former refers to hemorrhage treatment caused by thrombocytopenia, while the latter is special for patients who have no bleeding but display a severe platelet reduction. Hemorrhages caused by panhematopenia may occur in patients with haematological diseases when the patient's condition deteriorates after high-dose chemotherapy and/or radiotherapy. Platelet transfusion can reduce hemorrhaging caused by thrombocytopenia or platelet dysfunction. Most platelets are used for prophylactic transfusions, with the aim of reducing bleeding in thrombocytopenia patients. For a long time, the threshold of prophylactic infusion has been set for $20 \times 10^{9} / \mathrm{L}$. Many clinical researches have discussed how to reduce this threshold in the last decade. Researchers have analyzed the treatment outcome and 
other effects of the prophylactic infusion threshold. These studies indicate that it is safe to use $10 \times 10^{9} / \mathrm{L}$ as the threshold for patients in a stable condition and having no significant risk factors ${ }^{[21]}$. An appropriate reduced transfusion threshold may decrease platelet transfusion time to a certain extent, thus reducing the probability of the isoimmunity.

\section{Assistant treatment of platelet transfusion}

Patients that receive platelet transfusion, especially those with hematopathy, are often administered hormone, intravenous immunoglobulin, recombinant human thrombopoietin (TPO) to enhance the theraputical effect of platelet transfusion or reduce its dosage. Liu et $a l^{[23]}$ discussed the therapeutic effect of combination of anti thymocyte globulin (ATG) and Cyclosporin A (CsA) to assist patients with severe aplastic anemia (SAA), they concluded that the platelets transfusion interval can be appropriately extended after ATG and CsA treatment for 1 month. A prospective study by Zhao et $a l^{[24]}$ showed that a single application of small dose IVIG can elevate platelets by nearly $50 \%$, demonstrating that children with acute ITP were able to attain a relatively safe platelet count range, thereby avoiding intracranial bleeding and/or other life-threatening bleeding. Other researches suggest that for patients with severe sepsis, malignant tumor, renal anemia, and thrombocytopenia after radiation and chemotherapy, recombinant human thrombopoietin (rhTPO) showed good progress during clinical treatment ${ }^{[25-28]}$. RhTPO can also promote the survival of platelets after hematopoietic stem cell transplantation ${ }^{[29]}$. In addition, Guan et $a l^{[30]}$ and other researchers have shown that leukocyte poor APs can effectively improve the clinical effect of patients with hematopathy, and reduce the incidence of NHFTR significantly.

\section{Other}

The patient's own immune factors are also important causes of PTR, such as infection, hemorrhage, hypersplenism, DIC, drug treatment, and so on ${ }^{[31]}$. Dosage, storage time, and type of the platelets are also significant. Thus, it is important to pay close attention to the patient's condition and conduct platelet infusion rationally to decrease the occurrence of PTR. The effects on clinical outcomes of intraoperative platelet transfusion have been widely reported. A study of patients with acute type A aortic dissection suggested that intraoperative platelet transfusion did not improve the prognosis of hospitalized patients with aortic arch replacement ${ }^{[32]}$. For patients with intraoperative platelet transfusion, the incidence of postoperative sternal wound infection has increased.

\section{MOLECULAR MECHANISMS OF PTR}

\section{Relationship between platelet-related fac- tors and transfusion curative effects}

Platelet activation plays a very important role in the occurrence of hematology disease. Abnormal activation may cause loss of platelet adhesion function and aggregation in vivo, thus influence the hemostatic effect and curative effect. Studies have shown that platelet related factors are closely related to platelet activation. Liu et al ${ }^{[33]}$ have demonstrated that platelet activating factor (PAF) CD62P and CD63 are the influence factors of thrombus formation in the coronary artery and in early stage of myocardial infarction in acute coronary syndrome (ACS), and the determination of PAF can make important clinical sense in monitoring the curative effect and accessing the prognosis. Li et al ${ }^{[34]}$ have reported that antiplatelet drugs like glaucocalyxin A can inhibit the activation of platelets. The activity of tissue factor is closely related to platelet activation, and some scholars believe that tissue factor microparticles released from platelets or other cells may be the main active form of tissue factor in serum ${ }^{[35]}$.

TPO is the main regulator in megakaryocytes proliferation, maturation and platelet production ${ }^{[36]}$. Liu's group ${ }^{[37]}$ has detected the serum TPO level in thrombocytopenia patients and normal controls, and found that serum TPO level has a positive correlation with the degree of malignancy in hematologic diseases, and a negative correlation with the curative effect, and no correlation with secondary thrombocytopenia induced by other diseases. Platelet membrane glycoprotein (GP) also plays an important role in the platelet activation process. The polymorphism of GP can alter its antigenicity, and regulate its expression level and structure, thus affect the functional status of platelets and be closely related to hematologic diseases ${ }^{[38]}$.

The activity PAFs in patients not only participate in the whole process of disease occurrence and development by influencing the quantity and functional status of platelets, but are also involved in the regulation of newly imported platelets by affecting the activation and metabolic process. Thus, those factors are closely related to the curative effect of platelets. Similarly, the activity levels of PAFs in donors is related to the quality of platelet products, therefore, we speculate that the levels of PAFs in products or their changes in the storage period may also influence the curative effect of platelets.

\section{Signaling pathways in platelet}

With the incidence of hematologic diseases like thrombocytopenia and platelet dysfunction rising 
year by year, researches on the platelet and activating signaling pathways are becoming increasingly important ${ }^{[39]}$. Platelet signal transduction can be divided into three processes: 1) Platelet activator combines the membrane receptor, and mediates the early signal transduction; 2) Each signal pathway mutually coordinates, and finally forms a common inside-out signaling pathway, which can activate the GP II b/ III a receptor; and 3) Activated GP II b/ III a receptor mediates an outside-in signaling pathway ${ }^{[40]}$. The currently known platelet signaling pathways mainly include phospholipase C- $\beta$ (PLC- $\beta$ ) pathway ${ }^{[41]}$, protein tyrosine kinase pathway phosphatidylinositol 3-kinase (PI3K) pathway ${ }^{[42]}$, mitogen-activated protein kinase (MAPK) pathway ${ }^{[43]}$, adenosine cyclic monophosphate - protein kinase A (cAMP-PKA) pathway ${ }^{[40]}$, etc.

\section{PLC $-\beta$ pathway}

PLC- $\beta$ isoforms hydrolyze phosphatidylinositol 4,5-trisphosphate (PIP2) generates the second messengers inositol 1,4,5-trisphosphate (IP3) and 1,2-diacylglycerol (DAG). The latter in turn releases intracellular calcium stores and activate classical and novel isoforms of protein kinase $\mathrm{C}$ (PKC), respectively. An inherited deficiency of PLC- $\beta 2$ has been reported to result in a bleeding diathesis and defective platelet aggregation and secretion ex vivo. Since PLC $-\beta 2$ is the predominant isoform of PLC $-\beta$ in human platelets, this suggests PLC $-\beta$ is a critical component of platelet signaling ${ }^{[44]}$.

\section{PI3K pathway}

PI3K phosphorylates PIP2 to generate the lipid second messenger phosphatidylinositol 3,4,5-triphosphates (PIP3). The role of PIP3 in platelets remains to be identified, but it does lead to the activation of Akt 5, and stimulates the activity of both PDKl and exchange factors for Rho family GTPases in other cells. Although, there are no reports of genetic abnormalities of PI3K gamma in humans, Hirsh and his colleagues reported that PI3K gamma-null mice had a mild platelet aggregation defect and impaired thrombosis following injection with collagen and epinephrine ${ }^{[45]}$.

\section{MAPK pathway}

The MAPK pathway includes many proteins activity, including MAPK (mitogen-activated protein kinases, originally called ERK, extracellular signalregulated kinases), which can add phosphate groups to a neighboring protein, which acts as an "on" or "off" switch. By altering the levels and activities of transcription factors, MAPK leads to altered transcrip- tion of genes that are important for cell cycle. MAPK signaling plays a critical role in the regulation of both cellular proliferation and differentiation. Studies have shown that MAPK pathway is activated during TPO stimulation $^{[46]}$.

The platelet signal transduction system is a complete network, and it works through related factors like PAFs and TPO. Platelet activator combines with its receptor to generate the primary signal, and adjust platelet activity and cause the deformation and aggregation of platelet adhesion, secretion of particle contents and clot retraction through the delicate coordination of cascade of signal pathways. Further researches elucidating the platelet signaling pathways could help to reveal the molecular mechanism of platelet transfusion and its efficacy.

\section{CONCLUSION}

At present, platelet transfusion is an important clinical therapeutic method in thrombocytopenia. Studies on the preparation and preservation methods of platelets affect the potential quality of platelets, and the establishment of known HPA type platelet donor banks could help to improve the clinical efficacy of platelet transfusion, and reduce the incidence of platelet transfusion. Presently, domestic and overseas scholars still lack a comprehensive and clear understanding of platelet signal transduction mechanisms. Further studies focusing on platelet activation and signal transduction mechanisms, are important not only to illustrate the pathophysiology of platelets, but also to provide a theoretical guideline to the development of new anti-platelet drugs.

\section{References}

[1] Wei Q, Tian Z. The clinical application of platelets. Chin J Blood Transfusion, 2008; 21(9): 732-734.

[2] Han X, Li D. Research review of platelet transfusion resistance. Journal of Military Surgeon in Southwest China, 2009; 11(2): 262-264.

[3] Zhao SM. The research progress of preparation and clinical application of platelet. Clin J Blood Transfusion,2008; 21(9): 728-731.

[4] Wang D, Zhang X, Guan Zh, et al. Retrospective analysis of the effectiveness of apheresis platelets transfusion in patients with haematological diseases. Chin J Blood Transfusion, 2008; 21(4): 248-249.

[5] Hao B, Lv, Y, Shao Sh, et al. Comparison the quality of platelet concentrate and apheresis platelet. Henan Medical Research, 2010; 19(2): 173-175.

[6] Lv Y, Hao B, Wang Y, et al. Comparison the quality and curative effect of platelet concentrate and apheresis platelet. Chin J Blood Transfusion, 2008; 21(9): 690-691. 
[7] Cao Y, Guo X, Guan X. Analysis of the clinical effects of frozen platelets. Journal of Clinical Transfusion and Laboratory Medicine, 2004; 6(3): 213.

[8] Zhao Y, Ren W, Gao Y, et al. The quality and curative effect of frozen apheresis platelets after preserved for 6 months. Journal of Clinical Hematology, 2010; 23(2): 116-117.

[9] Ding G. Cryopreservation of Platelets after Storage at Normal Temperature for 3 Days and Its Clinical Practice. Journal of Experimental Hematology, 2008; 16( 5): 1196- 1200.

[10] Fu L, Zhang J, Sun Ch, et al. Construction of eukaryotic expression vector of recombinant human platelet CD36 gene and protein expression. Chin J Blood Transfusion, 2012; 25(06): 557-560.

[11] Chen Ch, Xue H, Zhou X, et al. Observation on the effect of HLA-I class matching paltelet transfusion on children with blood disease or undergoing hematopoietic stem cell transplantation. China Tropical Medicine, 2008; 8(10): 1713-1726.

[12] Zhao T. Research overview of human platelet antigen (HPA). Chin J Blood Transfusion, 2004; 17(2): 129-132.

[13] Sun G, Duan X, Zhang Y, et al. Analysis of Genetic Polymorphism in Randomized Donor's HPA 1-16 Antigens and Establishment of Typed Platelet Donor Data Bank. Journal of Experimental Hematology, 2005; 05:889-895.

[14] Wu Q, Liu M, Qi J, et al. Gene and Haplotype Frequencies for the Loci HLA-A, B and DRB1 in 11755 North Chinese Han Bone Marrow Registry Donors. Journal of Experimental Hematology, 2007; 15( 2) : 357-363.

[15] Che J. Discussion of platelet matching methods and establishment of blood donors HPA gene banks. Chin J Blood Transfusion (supplement), 2010; 23: 138.

[16] Shen W, Li L, Liao Y, et al. Multiple PCR-SSP technique for HPL-1-17 genotyping and HPA genotyping polymorphism distribution of Zhuang population in Guangxi. Chin J Blood Transfusion, 2014; 27(1): 8-14.

[17] Jiao Sh, Liu X, Chi X, et al. HPA-1-5-15 polymorphism distribution of Han population in Qingdao. Chin J Blood Transfusion, 2008; 3(21): 179-182.

[18] Chen Y, Ye X, Xia W, et al. Genetic polymorphism of HPA-1 to-17 alloantigen system in Guangzhou population. Chinese Journal of Immunology, 2010; 26(8): 699702

[19] Xu X, Liu Y, Ying Y, et al. Human platelet antigen allele frequencies and new mutations on platelet glycoprotein genes in the Chinese Han population. Transfus Med, 2011; 21(5): 330-7.

[20] Li RS, Qian ZL, Ling B, Lu P. Establishment of reference panel for human platelet antigen genotyping. Vox Sang, 2014; 107(2): 166-70.

[21] Liu Zh, Wang Zh, Zhang Y, et al. Application of HPA matching in immune ineffective platelet transfusion. Chin J Blood Transfusion, 2011; 24(6): 474-477.

[22] Liu B, Gao G, Wang D, et al. HPA Distribution Characteristics of Platelet Donor Population in Mudanjiang
Area of China and Establishment of Its Database. Journal of Experimental Hematology, 2012; 20(3) : 757-761.

[23] Liu J, Li X, Guo N, et al. The therapeutic effects of platelets transfusion in 21 patients with severe aplastic anemia after intensive immunosuppressive therapy. Chinese Journal of Practical Internal Medicine, 2012; 32(5): 374-375.

[24] Zhao W, Hua Y, Lv X, et al. Study of efficacy of single low-dose intravenous immunoglobulin in elevating platelet counts. Chinese Journal of Practical Pediatrics, 2012; 27(11): 863-865.

[25] Yu D, Zhang N, Cheng H, et al. Recombinant human thrombopoietin in treatment of chemotherapy-induced thrombocytopenia in patients with acute leukemia. Journal of Clinical Medicine in Practice, 2013; 17(17): 113-115.

[26] Chen J, Qu Q, Wei Y, et al. Comparison of effect between rhTPO and rhIL-11 on treatment for cancer patients with thrombocytopenia. Journal of Practical Oncology, 2010; 25(3): 318-320.

[27] Wu Q, Ren J, Wu X, et al. Recombinant human thrombopoietin improves platelet counts and reduces platelet transfusion possibility among patients with severe sepsis and thrombocytopenia: a prospective study. J Crit Care, 2014; 29(3): 362-6.

[28] Shi H. The effect of rhTPO on platelets in patients with chronic renal failure-induced anemia. Chin J Hematology, 1997; 11: 39.

[29] Han tt, Xu LP, Liu DH, et al. Recombinant human thrombopoietin promotes platelet engraftment after haploidentical hematopoietic stem cell transplantation: a prospective randomized controlled trial. Ann Hematol, 2015; 94(1): 117-28.

[30] Guan X, Yu Y, Feng Q, et al. Analysis of the Effectiveness of Leucocyte-depleted Apheresis Platelets Transfusionin Patients with Haematological Diseases. Science Technology and Engineering, 2010; 10(7): 1747-1748.

[31] Lv Y, Sun G, Zhang Y, et al. The Impact of Different Threshold of Prophylactic Platelet Transfusion on $\mathrm{Pa}-$ tients with Hematologic Disease. Labeled Immunoassays and Clinical Medicine, 2015; 22(2): 89-91.

[32] Wu B, Wang Y, Wang C, et al. Intraoperative platelet transfusion is associated with increased postoperative sternal wound infections among type A aortic dissection patients after total arch replacement. Transfus Med, 2014; 24(6): 400-5.

[33] Liu K, Wei Y, Li J. Expression of platelet-activating factor with acute coronary syndrome. Journal of Shandong Medical College, 2013; 35(1): 15-16.

[34] Li W, Tang X, Yi W, et al. Glaucocalyxin A inhibits platelet activation and thrombus formation preferentially via GPVI signaling pathway. PLoS One, 2013; 8(12): e85120.

[35] Huang X, Chen F. The research progress on plateletassociated tissue factor. Journal of Central South University(Medical Science), 2005; 30(2): 233-235.

[36] Liu H, Cong Y, Mao B. Progress in study of receptor 
signaling pathways induced by thrombopoietin. Bulletin of The Academy of Military, 2005; 29(5): 471-474.

[37] Liu W, Wu X, Li F, et al. The significance of serum thrombopoietin levels in patients with thrombocytopenic diseases[J]. Medical Journal of Chinese People's Health, 2008; 20(9): 900-902.

[38] Liu P, Zhao Y. The relationship of platelet membrane glycoproteins polymorphism and thrombogenesis. Chinese Journal of Thrombosis and Hemostasis, 2003; 9(4): 178-181.

[39] Tang X, Chen F, Qian Z, et al. Effect of the defect of integrin $\alpha$ II b $\beta 3$ on the inside-out signal transduction in platelets. Journal of Central South University (Medical Science), 2002; 27(3): 207-210.

[40] Lu S, Meng Zh. Research Progress of Platelet Membrane Protein Receptor Signal Transduction Pathways. Medical Recapitulate, 2014; 20(21): 3851-3854.

[41] Zheng Y, Adams T, Zhi H, et al. Restoration of responsiveness of phospholipase $\mathrm{C} \gamma$ 2-deficient platelets by enforced expression of phospholipase $\mathrm{C} \gamma$ 1. PLoS One, 2015; 10(3): e0119739.

[42] Shi C, Zhu X, Wang J, et al. Tanshinone IIA promotes non-amyloidogenic processing of amyloid precursor protein in platelets via estrogen receptor signaling to phosphatidylinositol 3-kinase/Akt. Biomed Rep, 2014; 2(4): 500-504.

[43] Yu L, Huang X, Huang K, et al. Ligustrazine attenuates the platelet-derived growth factor-BB-induced proliferation and migration of vascular smooth muscle cells by interrupting extracellular signal-regulated kinase and P38 mitogen-activated protein kinase pathways. $\mathrm{Mol}$ Med Rep, 2015; 12(1): 705-711.

[44] Lian L, Wang Y, Draznin J, et al. The relative role of PLC $\beta$ and PI3K $\gamma$ in platelet activation. Blood, 2005; 106(1):110-117.

[45] Hirsch E, Bosco O, Tropel P, et al. Resistance to thromboembolism in PI3K-deficient mice. Faseb J, 2001; 15: 2019-2021.

[46] Lannutti BJ, Drachman JG. Lyn tyrosine kinase regulates thrombopoietin-induced proliferation of hematopoietic cell lines and primary megakaryocytic progenitors. Blood, 2004; 103(10): 3736-3743.

(Received 30 December 2016, Revised 13 February 2017, Accepted 01 March 2017) 